Partial discharges within two spherical voids in an epoxy resin

This article has been downloaded from IOPscience. Please scroll down to see the full text article.

2013 J. Phys. D: Appl. Phys. 46335301

(http://iopscience.iop.org/0022-3727/46/33/335301)

View the table of contents for this issue, or go to the journal homepage for more

Download details:

IP Address: 152.78.191.137

The article was downloaded on 05/09/2013 at 08:58

Please note that terms and conditions apply. 


\title{
Partial discharges within two spherical voids in an epoxy resin
}

\author{
H A Illias ${ }^{1,2}$, G Chen ${ }^{3}$, A H A Bakar ${ }^{2}$, H Mokhlis ${ }^{1,2}$ and M A Tunio ${ }^{1}$ \\ ${ }^{1}$ University of Malaya High Voltage Laboratory, Department of Electrical Engineering, \\ Faculty of Engineering, University of Malaya, 50603 Kuala Lumpur, Malaysia \\ ${ }^{2}$ UM Power Energy Dedicated Advanced Centre (UMPEDAC), Level 4, Wisma R\&D UM, \\ University of Malaya, Jalan Pantai Baharu, 59990 Kuala Lumpur, Malaysia \\ ${ }^{3}$ Tony Davies High Voltage Laboratory, University of Southampton, Southampton, SO17 1BJ, UK \\ E-mail: h.illias@um.edu.my
}

Received 4 April 2013, in final form 14 June 2013

Published 29 July 2013

Online at stacks.iop.org/JPhysD/46/335301

\begin{abstract}
A void in a dielectric insulation material may exist due to imperfection in the insulation manufacturing or long term stressing. Voids have been identified as one of the common sources of partial discharge (PD) activity within an insulation system, such as in cable insulation and power transformers. Therefore, it is important to study PD phenomenon within void cavities in insulation. In this work, a model of PD activity within two spherical voids in a homogeneous dielectric material has been developed using finite element analysis software to study the parameters affecting PD behaviour. The parameters that have been taken into account are the void surface conductivity, electron generation rate and the inception and extinction fields.

Measurements of PD activity within two spherical voids in an epoxy resin under ac sinusoidal applied voltage have also been performed. The simulation results have been compared with the measurement data to validate the model and to identify the parameters affecting PD behaviour. Comparison between measurements of PD activity within single and two voids in a dielectric material have also been made to observe the difference of the results under both conditions.
\end{abstract}

(Some figures may appear in colour only in the online journal)

\section{Introduction}

For many years, modelling of a partial discharge (PD) event within a void cavity in a dielectric material has been an area of active research in insulation diagnosis and performance assessment of an insulation system. This is because PD in a void is one of the major factors that causes insulation damage in high-voltage equipment, such as in cable insulation and power transformers. Most PDs are commonly detected in voids within solid insulation through pulses induced in an external circuit [1]. A void in a dielectric insulation is a gas-filled cavity which exists due to imperfection during the insulation manufacturing. During curing of the insulation material, air can leak into the mould due to the insufficient pressure on the material, causing vapour pressure of the material component to develop a gas-filled void [2]. On the other hand, voids may be formed during the working life of the insulation. Void cavity is one of the potential sources of PD activity in a dielectric insulation, which may degrade the insulation system leading to insulation failure [3].

In reality, void shapes that are most commonly found are ellipsoidal and spherical. In insulation, two or more voids which exist in a random position are more likely to be found. However, works on PD activity within more than one void in a dielectric are not widely published. Most of the published works reported on measurement and modelling of PD events within a single void in a dielectric material [4-11]. Therefore, it is interesting to study PD activity within more than one void in a dielectric material. The obtained results may be useful in condition monitoring and performance assessment of an insulation system.

In previous work, a numerical analysis of two-dimensional (2D) model geometry has been developed to study the electric field distribution within two voids in a dielectric material, where the voids were placed along the same horizontal and vertical axes of the applied field direction [12]. From the model, the maximum field in both voids was found to be 
lower when two voids are located along the same vertical axis of the applied field direction than along the same horizontal axis, resulting in a higher PD inception field. This agrees with their measurement results. However, the measured ac breakdown strength for this type of voids arrangement was found to be lower than that of a single void due to the reduction of the effective material thickness. Two voids which are positioned along the same horizontal axis of the applied field direction yields the same PD characteristics with single void in a dielectric. Further distance between the two voids does not significantly affect the field in each void.

Another 2D model geometry has also been developed to study the maximum electric field magnitude within more than one cylindrical void in a dielectric material for different void locations $[13,14]$. The field in the model was determined using boundary element method, where charge distribution on each boundary is estimated so that it produces a field that satisfies those imposed boundary conditions. It was found that the maximum field in each void is also lower when all voids are located along the same vertical axis of the applied field direction compared to along the same horizontal axis. When the number of voids increases along the same vertical axis of the applied field direction, the maximum field magnitude in each void becomes lower.

In this work, a three-dimensional (3D) model geometry of two spherical voids located in a homogeneous dielectric material has been developed using finite element analysis (FEA) software. The model has been used to study PD activity within two spherical voids in a dielectric insulation material. This model is an extension of the FEA model from the previous published papers $[11,15,16]$. Measurements of PD activity within two spherical voids in an epoxy resin under ac sinusoidal applied voltage have also been carried out. Comparison between simulation and measurement results has been performed to validate the model and to identify the parameters affecting PD behaviour. The parameters affecting PD behaviour that can be readily identified from the model are the void surface conductivity, electron generation rate (EGR) and the inception and extinction fields.

\section{PD in voids}

For a PD to occur in a void, the electric field in the void must exceed the breakdown strength of the gas, i.e. the void inception field, $E_{\text {inc }}$ is exceeded and there must be a free initial electron existing to initiate an electron avalanche $[2,6,7]$. The main sources of initial free electron are surface emission, where a free electron is emitted from the void surface due to electric field and temperature, and volume ionization, where an electron appears through radiative gas ionization by an energetic photon $[6,7]$. When a PD occurs in a void, the void becomes conducting from that of non-conducting state [2,9]. During a PD event, the flow of current through the streamer due to electron avalanche causes the electric field in the void to decrease. Discharge stops once the field in the void becomes less than the extinction field [6,7].

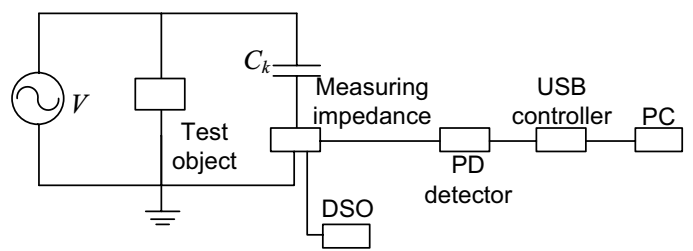

Figure 1. The measurement setup.

\section{PD measurement}

The PD measurement setup, as shown in figure 1, comprises a high-voltage supply, $V$, a coupling capacitor $C_{k}$, a test object, a measuring impedance (MI), a digital signal oscilloscope (DSO), a PD detector, a USB controller and a personal computer (PC) [16]. The DSO is connected to MI to capture PD signals as a function of time. The MI and PD detector detect the PD signals and are displayed on the PC. The PD detector was interfaced with its software in the PC. Therefore, noise was eliminated from the measurement by setting a threshold limit from the PD detector software. The PD pulses were only recorded when their magnitudes were higher than the threshold limit. All pulses below the threshold limit were eliminated.

The test object consists of a cylindrical epoxy resin which was connected with two electrodes on its top and bottom surfaces (figure 2). The top electrode was connected to a $50 \mathrm{~Hz}$ ac sinusoidal voltage of amplitude $U_{\text {app }}$ and the bottom electrode was grounded. The whole test object was immersed in mineral oil during the whole experiment. Initially, two bubbles of air were injected into an epoxy resin before it cured completely. Then, the cured resin containing the bubbles are cut into two smaller pieces. Within a larger volume of uncured epoxy resin, the two smaller pieces of the cured epoxy, where each containing a spherical void of diameters $d_{1}$ and $d_{2}$, were positioned next to each other with a gap, $g_{d 1-d 2}$, as shown in figure 2 . The smaller pieces were embedded into the uncured resin by using a tweezer, which held the smaller pieces and released them after they were placed at the desired positions. After that, the epoxy was cured for $24 \mathrm{~h}$ at ambient temperature, post cured for $4 \mathrm{~h}$ at $90^{\circ} \mathrm{C}$ and cooled down to ambient temperature for $2 \mathrm{~h}$.

The samples that have been prepared for the experiment are shown in table 1 . The aim of creating samples 1 and 2 is to compare PD events within two voids of same (sample 1) and different (sample 2) sizes, which are located very close to each other. Sample 3, which consists of one void in epoxy resin has also been prepared to compare the PD patterns from single and two voids samples.

\section{FEA model geometry}

The model geometry has been developed using FEA software in three-dimensional (3D) due to its non-axial-symmetric geometry. Figure 3 shows a 3D model geometry which consists of two spherical voids located within a dielectric material (10 $\mathrm{mm}$ radius and $4 \mathrm{~mm}$ thickness) and also the void surface (a layer of $0.05 \mathrm{~mm}$ thickness surrounding the void). The void surface was used to model the charge movement along the 


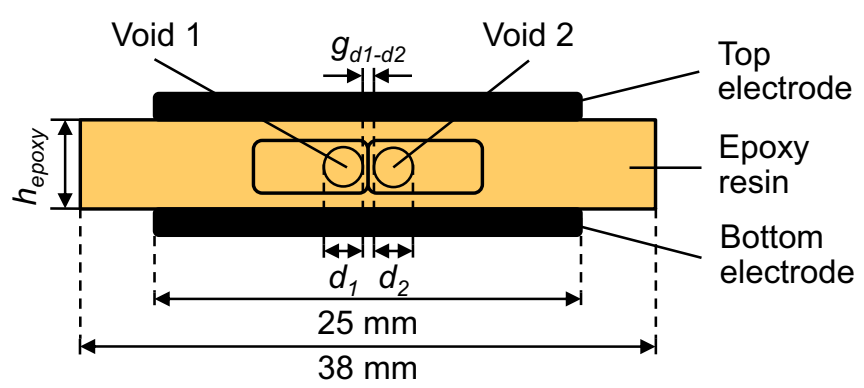

Figure 2. The test object.

Table 1. Dimensions of the samples that have been prepared.

\begin{tabular}{llllll}
\hline Sample & $\begin{array}{l}d_{1} \\
(\mathrm{~mm})\end{array}$ & $\begin{array}{l}d_{2} \\
(\mathrm{~mm})\end{array}$ & $\begin{array}{l}g_{d 1-d 2} \\
(\mathrm{~mm})\end{array}$ & $\begin{array}{l}h_{\text {epoxy }} \\
(\mathrm{mm})\end{array}$ & $\begin{array}{l}U_{\mathrm{app}} \\
(\mathrm{kV})\end{array}$ \\
\hline 1 & 2.7 & 2.7 & 0.6 & 4.0 & 20 \\
2 & 3.0 & 3.6 & 0.7 & 5.4 & 22 \\
3 & 2.7 & - & - & 4.0 & 14 \\
\hline
\end{tabular}

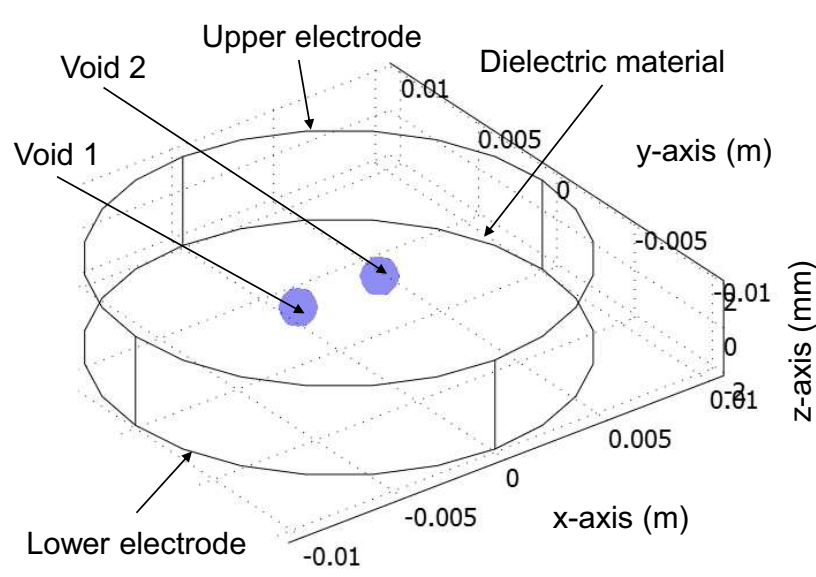

Figure 3. 3D model geometry for two voids located in the dielectric material.

void surface, which is explained in section 4.3. A $50 \mathrm{~Hz}$, ac sinusoidal voltage $\left(U_{\text {app }}\right)$ was applied to the upper electrode while the lower electrode was always grounded. The meshing of the model was refined twice so that more mesh elements are obtained, which will generate more accurate results. The number of the mesh elements is 60863 for sample 1 and 63310 for sample 2 .

The governing partial differential equation (PDE) to solve the electric field distribution in the model is

$$
-\nabla \cdot(\sigma \nabla V)-\varepsilon \nabla \cdot(\partial \nabla V / \partial t)=0
$$

where $V$ is the electric potential, $\sigma$ is the conductivity and $\varepsilon$ is the permittivity.

\subsection{Statistical modelling of PD occurrence}

There are two conditions which must be fulfilled before a PD can occur in each void; the electric field in void $x$ must be higher than the inception field in the void, $E_{\text {inc } x}$, and there must be an initial free electron available in the void to start a
PD. The inception field (in $\mathrm{V} \mathrm{m}^{-1}$ ) is calculated using [6, 7]

$$
E_{\mathrm{inc} x}=(E / p)_{\mathrm{cr}} p_{x}\left(1+B / \sqrt{2 p_{x} r_{\mathrm{v} x}}\right),
$$

where $r_{\mathrm{v} x}$ is the void $x$ radius (in $\mathrm{m}$ ), $p_{x}$ is the pressure in void $x$ (in Pa) and $(E / p)_{\text {cr }}$ and $B$ characterize the ionization processes in the gas. For air, it has been defined that $(E / p)_{\mathrm{cr}}$ is $25.2 \mathrm{~V} \mathrm{~Pa}^{-1} \mathrm{~m}^{-1}$ and $B$ is $8.6 \mathrm{~m}^{1 / 2} \mathrm{~Pa}^{1 / 2}$.

The availability of an initial free electron will determine the statistical distributions of the PD pattern, such as phase and charge magnitude distributions. The initial free electron availability is modelled by calculating the total EGR, which represents the total number of free electrons generated per unit time. The total EGR in void $x$ (where $x$ equals to 1 and 2), $N_{\text {et } x}$ is calculated using [16]

$$
N_{\mathrm{et} x}(t)=N_{\mathrm{es} x}(t)+N_{\mathrm{ev} x},
$$

where $N_{\mathrm{es} x}(t)$ is the EGR due to surface emission and $N_{\mathrm{ev} x}$ is the EGR due to volume ionization in void $x . N_{\mathrm{ev} x}$ is assumed to be time independent and always available because it is due the radiation ionization occurring in the void.

The time dependent $N_{\mathrm{es} x}(t)$ is defined as [16]

$N_{\mathrm{es} x}(t)=N_{\mathrm{PD} x} \exp \left[-\left(t-t_{\mathrm{PD} x}\right) / \tau_{\mathrm{dec} x}\right] \exp \left|E_{\mathrm{v} x}(t) / E_{\mathrm{inc}} x\right|$,

where $t_{\mathrm{PD} x}$ is the time elapsed since previous PD has occurred in void $x$ and $\tau_{\operatorname{dec} x}$ is the charge decay time constant. $N_{\mathrm{PD} x}$ equals to

$$
N_{\mathrm{PD} x}=N_{\mathrm{es} 0 x}\left|E_{\mathrm{v} x}\left(t_{\mathrm{PD} x}\right) / E_{\mathrm{inc} x}\right|,
$$

where $N_{\mathrm{es} 0 x}$ is called the initial EGR due to surface emission when $E_{\mathrm{v} x}\left(t_{\mathrm{PD} x}\right)$ equals $E_{\mathrm{inc} x}$. Since $E_{\mathrm{inc} x}$ is assumed to be constant throughout the simulation, $N_{\mathrm{es} 0 x}$ is also constant. $N_{\mathrm{PD} x}$ represents the initial EGR due to surface emission for $E_{\mathrm{v} x}(t)$ at $t_{\mathrm{PD} x}$ of the previous PD occurrence. Since $N_{\mathrm{es} x}(t)$ (in (4)) depends on the previous PD occurrence, the total EGR in (3) for the first PD occurrence only depends on $N_{\mathrm{ev} x}$. Equations (4) and (5) have been explained in detail in a previous publication [16].

It is common to model the probability of a PD occurrence in each void, $P_{x}$ which depends on a random number, $R_{x}$ $\left(0<R_{x}<1\right)$. $P_{x}$ is calculated using

$$
P_{x}(t)=N_{\text {et } x}(t) \Delta t
$$

where $\Delta t$ is the time-stepping interval when no PD occurs. A PD will only occur when $E_{\mathrm{v} x}>E_{\mathrm{inc} x}$ and $P_{x}>R_{x}$.

\subsection{PD events and PD charge magnitude}

In order to reduce the calculations and to simplify the model, a PD in the void is assumed to affect the whole void space. A PD is also assumed to occur only along the symmetry axis. Therefore, a PD is modelled by changing the value of the void conductivity from zero to a high value, $\sigma_{\mathrm{vH}} \cdot \sigma_{\mathrm{vH}}$ is a value which can cause a PD to last for few nanoseconds. When a PD occurs, the electric field in the void at time $t, E_{\mathrm{v} x}(t)$ decreases and PD stops when $E_{\mathrm{v} x}(t)$ becomes less than the 
extinction field, $E_{\text {ext } x}$. During a PD occurrence, the timestepping interval, $\Delta t$ is changed to very small value to solve the PD event. This is because a large time-stepping interval will cause the electric field in the void to drop very quickly during a PD, resulting in the PD stopping at a level far below the extinction field. After a PD has completed, the time-stepping interval is changed back to its original value, i.e. during no PD event. The real and apparent PD change magnitudes in each void, $q_{\text {real } x}$ and $q_{\text {app } x}$, are calculated by integrating the current through the void and upper electrode surfaces with time during PD occurrence,

$$
\begin{gathered}
q_{\text {real } x}=\int_{t}^{t+\Delta t} J_{\mathrm{v} x}(t) S_{\mathrm{v} x} \mathrm{~d} t, \\
q_{\text {app } x}=\int_{t}^{t+\Delta t} J_{\text {elec }}(t) S_{\text {elec }} \mathrm{d} t,
\end{gathered}
$$

where $J_{\mathrm{v} x}(t)$ and $J_{\text {elec }}(t)$ are the current density through the void and upper electrode surface during a PD occurrence and $S_{\mathrm{v} x}$ and $S_{\text {elec }}$ are the surface area in the middle of the void and the upper electrode, respectively.

\subsection{Charge decay through surface conduction}

After a PD has occurred, some charges that remain free along the void surface may move freely along the void surface. It is assumed that the charge movement depends on the polarity of the electric field in the void, $E_{\mathrm{v} x}(t)$ and electric field due to surface charge, $E_{\mathrm{q} x}(t)$ [11]. When the polarity of $E_{\mathrm{v} x}(t)$ is the same with $E_{\mathrm{q} x}(t)$, the charges are assumed to move away from the centre of the void. This phenomenon is modelled by increasing the void surface conductivity, $\sigma_{\mathrm{s} x}$ from a lower value to a higher value, $\sigma_{\mathrm{sH} x}$. When the polarity of $E_{\mathrm{v} x}(t)$ is the opposite of $E_{\mathrm{q} x}(t)$, the charges are assumed not to be moving. The void surface conductivity is maintained or reset to its lower value.

\subsection{Parameters in the model}

Table 2 defines all parameters that have been used in the simulation. Any parameters in this table with values are used in the simulation for samples 1 and 2. The choice of these parameter values is detailed in [16]. However, parameters without values in this table are dependent on the samples that have been prepared, which is explained in the results and discussion section.

The values of the parameters in table 2 were chosen based on the comparison between measurement and simulation results. Sensitivity analysis was used to select the values of $N_{\mathrm{es} 0 x}$ and $N_{\mathrm{ev} x}$. The mean square error (MSE) between simulation and measurement results in terms of the number of PDs per cycle versus the PD phase occurrence distribution, $H_{n}$, and the total charge per cycle versus the PD phase occurrence distribution, $H_{\mathrm{q} s}$, were calculated for different combinations of $N_{\mathrm{es} 0 x}$ and $N_{\mathrm{ev} x}$. The combination which gives the smallest MSE was chosen. The criteria used to choose these parameters are the difference between measurement and simulation of the number of PDs per cycle is \pm 0.1 and the total charge per cycle or maximum PD charge magnitudes is $10 \%$.
Table 2. Definition of parameters used in the simulation.

\begin{tabular}{llll}
\hline Definition & Symbol & Value & Unit \\
\hline Applied frequency & $f$ & 50 & $\mathrm{~Hz}$ \\
Time step during no PD & $\Delta t$ & $1 / 500 f$ & $\mathrm{~s}$ \\
Time step during PD & $\Delta t$ & 1 & $\mathrm{~ns}$ \\
Simulation cycles & $c y c$ & 500 & \\
Material relative permittivity & $\varepsilon_{\mathrm{rmat}}$ & 4.4 & \\
Void surface permittivity & $\varepsilon_{\mathrm{rs} x}$ & 4.4 & \\
Void relative permittivity & $\varepsilon_{\mathrm{rv} x}$ & 1 & \\
Material conductivity & $\sigma_{\mathrm{mat}}$ & $1 \times 10^{-13}$ & $\mathrm{Sm}^{-1}$ \\
Void conductivity during no PD & $\sigma_{\mathrm{v} 0 x}$ & 0 & $\mathrm{Sm}^{-1}$ \\
Void conductivity during PD & $\sigma_{\mathrm{vH} x}$ & $5 \times 10^{-3}$ & $\mathrm{Sm}^{-1}$ \\
Lower void surface conductivity & $\sigma_{\mathrm{sL} x}$ & $1 \times 10^{-13}$ & $\mathrm{Sm}^{-1}$ \\
Effective charge decay time & $\tau_{\mathrm{dec} x}$ & 2 & $\mathrm{~ms}$ \\
$\quad$ constant & & & \\
Material thickness & $h_{\mathrm{mat}}$ & & $\mathrm{mm}$ \\
Applied voltage & $U_{\mathrm{app}}$ & & $\mathrm{kV}$ \\
Inception field & $E_{\mathrm{inc} x}$ & & $\mathrm{kVmm}^{-1}$ \\
Extinction field & $E_{\mathrm{ext} x}$ & & $\mathrm{kVmm}^{-1}$ \\
Higher void surface conductivity & $\sigma_{\mathrm{sH} x}$ & & $\mathrm{Sm}^{-1}$ \\
Initial EGR due to surface & $N_{\mathrm{es} 0 x}$ & $\mathrm{~s}$ \\
emission & $N_{\mathrm{ev} x}$ & & $\mathrm{~s}$ \\
EGR due to & & & \\
volume ionization & & & \\
\hline
\end{tabular}

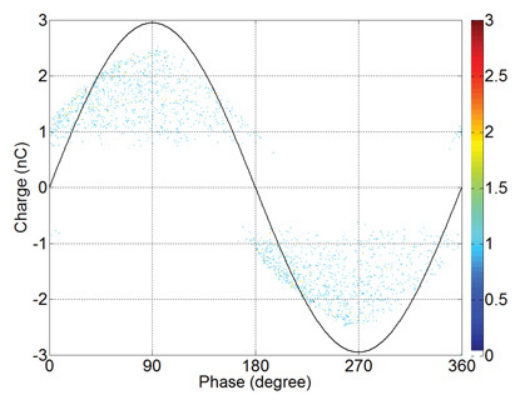

(a)
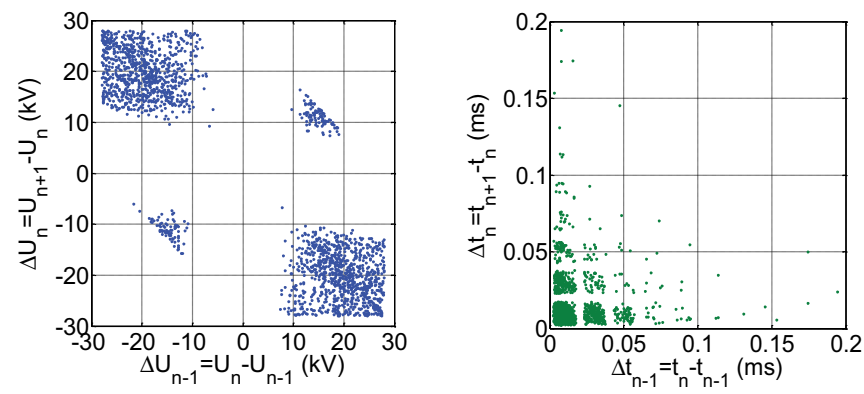

Figure 4. Measurement results of PD activity in single void (sample 3); (a) PRPD pattern and (b) PSA scatter plots.

\section{Results and discussion}

The results section is divided into two parts; comparison between measurement and simulation results that have been performed for two different samples and the electric field distributions and plots from the FEA model. The measurement results that have been obtained are presented in phaseresolved partial discharge (PRPD) histograms, PD phase distributions and pulse sequential analysis (PSA) scatter plots. Measurement of 1500 applied voltage cycles were recorded 
Table 3. PD data from the measurement and simulation for sample 1.

\begin{tabular}{lllll}
\hline & & \multicolumn{3}{c}{ Simulation } \\
\cline { 3 - 5 } Data & Measurement & Void 1 + Void 2 & Void 1 & Void 2 \\
\hline Total PDs per cycle & 9.6 & 9.6 & 8.9 & 0.7 \\
Total charge per cycle (pC) & 7550 & 8431 & 6872 & 1559 \\
Mean charge $(\mathrm{pC})$ & 786 & 881 & 772 & 2327 \\
Maximum charge, $q_{\max }(\mathrm{pC})$ & 3814 & 3758 & 2746 & 3758 \\
Minimum charge, $q_{\min }(\mathrm{pC})$ & 710 & 716 & 716 & 716 \\
\hline
\end{tabular}

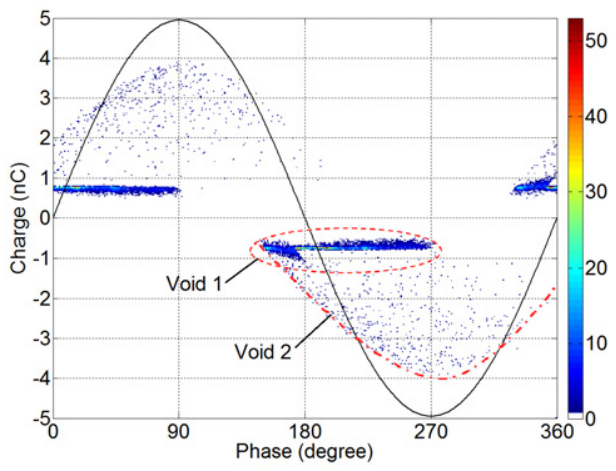

(a)

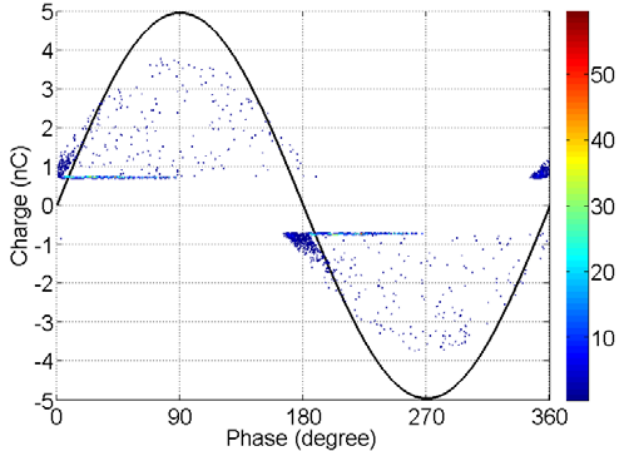

(b)

Figure 5. PRPD histograms from sample 1; $(a)$ measurement and $(b)$ simulation.

to ensure enough data were obtained. Measurement results from a single void have also been included to compare with the results obtained from two-void samples.

\subsection{Measurement results of PD activity within single void (sample 3)}

The measurement results of PD activity within a sample of single void in an epoxy resin that has been prepared are shown in figure 4. Figures 4(a) and (b) show PRPD pattern and PSA scatter plots from measurement of PD activity within single void in an epoxy resin (sample 3 ). The PSA scatter plots consist of time difference $(\Delta t)$ and applied voltage amplitude difference $(\Delta U)$ between consecutive discharges, where $n$ is the $n$th PD event [18]. The explanation for single void is not included in this paper because it has been detailed in previous publications $[11,16]$. The results are inserted for comparison between PD activity within single and two voids.

\subsection{Comparison between measurement and simulation results: sample 1}

The PRPD histogram of PD events obtained from PD measurement using sample 1 in table 1 is shown in figure 5(a). The red-dashed lines show a segregation of PD patterns from two different voids, where one of the voids is assumed to be void 1 and the other is void 2. Sample 1 is the test object which has two spherical voids of same size embedded into an epoxy resin. It can be seen that the number of PDs occurring in void 1, i.e. concentrated patterns at a certain area, is higher than in void 2, i.e. more dispersed patterns. This is different from the PRPD pattern for single void sample, which has only one common pattern. To study the factors which contribute to this behaviour, the measurement pattern has been reproduced by the simulation model. The measurement and simulation of PD patterns and data shown in table 3 can be said to be within reasonable agreement to each other although the pattern cannot be reproduced exactly.

Referring to figure $5(a)$, the number of PD is higher in void 1 than void 2 . This is due to the EGR is higher in void 1, resulting in larger probability of PD to occur. Most of PDs occur immediately after the inception field has been exceeded. Therefore, within one cycle of the applied voltage, more PDs occur, which can be seen by the high density of PD distribution pattern. However, in void 2, lower EGR reduces the probability of PD occurrence. Hence, most of PDs occur after certain time delay when the inception field has been exceeded. Thus, there is lower number of PDs per cycle, which can be seen by the low density of PD distribution pattern.

The PRPD patterns shown in figure $5(a)$ can also be represented using the phase distributions shown in figure 6, which are commonly used in PD pattern representation [17]. From the number of PDs per cycle versus phase distribution $\left(H_{n}\right)$, most PDs occur at the rising edge of the applied voltage but fewer PDs occur at the end of the falling edge due to more PDs originated from void 1 . The total charge per cycle versus phase distribution or $H_{\mathrm{qs}}$ has a right-skewed distribution. Since more PDs occur at the earlier part of the rising edge than the peak of the applied voltage, the total charge per cycle is also higher over that region. The mean charge versus phase distribution, $H_{\mathrm{q} n}$ is obtained from the ratio between $H_{\mathrm{qs}}$ and $H_{n}$. The mean charge is higher in the second and fourth quadrants of the applied voltage because fewer PDs per cycle with larger charge magnitude occur in those quadrants. From $H_{\mathrm{qm}}$ or the maximum charge versus phase distribution, the maximum charge magnitude is largest at the peak applied 


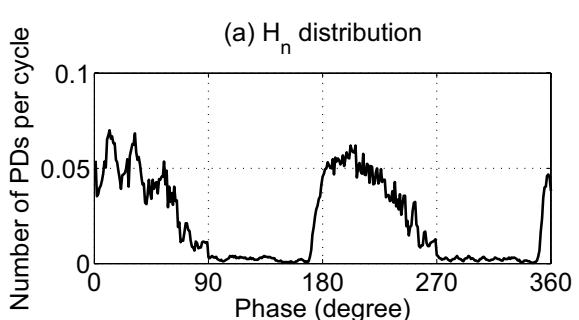

(c) $\mathrm{H}_{\text {qn }}$ distribution

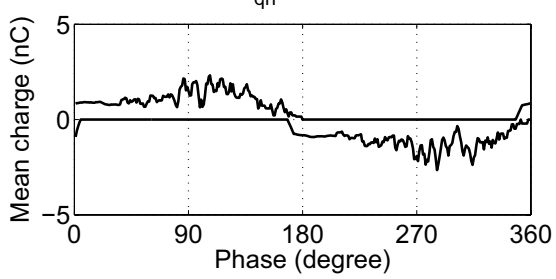

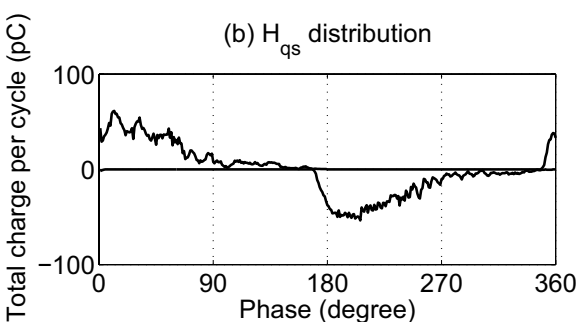

(d) $\mathrm{H}_{\mathrm{qm}}$ distribution

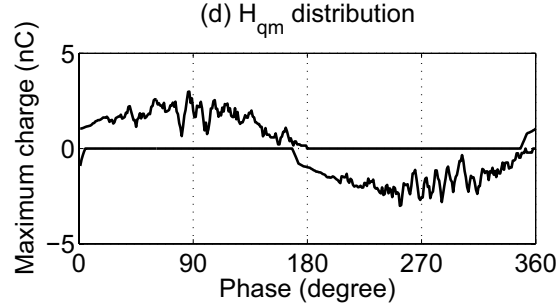

Figure 6. Phase distribution of PD activity measurement from sample 1.

Table 4. Simulation parameters used in the model for sample 1.

\begin{tabular}{|c|c|c|c|c|}
\hline Definition & Symbol & Void 1 & Void 2 & Unit \\
\hline Void diameter & $r_{\mathrm{v} x}$ & 2.7 & 2.7 & $\mathrm{~mm}$ \\
\hline Material thickness & $h_{\text {mat }}$ & 4 & 4 & $\mathrm{~mm}$ \\
\hline Applied voltage & $U_{\text {app }}$ & 20 & 20 & $\mathrm{kV}$ \\
\hline $\begin{array}{l}\text { Higher void surface } \\
\text { conductivity }\end{array}$ & $\sigma_{\mathrm{sH} x}$ & $1 \times 10^{-13}$ & $5 \times 10^{-13}$ & $\mathrm{Sm}^{-1}$ \\
\hline Inception field & $E_{\text {inc } x}$ & 3.69 & 3.69 & $\mathrm{kVmm}^{-1}$ \\
\hline Extinction field & $E_{\text {ext } x}$ & 1.8 & 1.8 & $\mathrm{kVmm}^{-1}$ \\
\hline $\begin{array}{l}\text { Initial EGR due to } \\
\text { surface emission }\end{array}$ & $N_{\mathrm{es} 0 x}$ & 30000 & 1 & $\mathrm{~s}^{-1}$ \\
\hline $\begin{array}{l}\text { EGR due to volume } \\
\text { ionization }\end{array}$ & $N_{\mathrm{ev} x}$ & 80 & 80 & $\mathrm{~s}^{-1}$ \\
\hline
\end{tabular}

voltage amplitude since the electric field in the void is at a maximum. These magnitudes are due to PDs from void 2.

Table 4 shows the parameter values that have been used in the simulation for sample 1 . The method of choosing the parameter values is mentioned in section 4.4. In order to reproduce a larger number of PDs per cycle in void 1 than void 2, the initial EGR due to surface emission, $N_{\mathrm{es} 0 x}$, is set higher for void 1 than void 2. However, the EGR due to volume ionization, $N_{\mathrm{ev} x}$ is the same because the volume of both voids is equal. The void surface conductivity during charge movement along the void surface, $\sigma_{\mathrm{sH} x}$ is set higher for void 2 than void 1 . This is because when $\sigma_{\mathrm{sH} x}$ is higher, the EGR due to surface emission, $N_{\mathrm{es} x}$ will decrease due to faster reduction of charge accumulation along the void surface. Thus, the probability of a PD occurrence, $P$ also decreases, reducing the number of PDs per cycle in void 2. This is why $\sigma_{\mathrm{sH} x}$ is set higher for void 2 than void 1 . The simulation data for voids 1 and 2 are shown in table 3 , where the simulated number of PDs per cycle is higher for void 1 than void 2 .

It is assumed that the initial pressure in the void is the same, which is the atmosphere pressure. Thus, the inception field, $E_{\text {inc } x}$ is the same for both voids. The extinction field, $E_{\text {ext } x}$ is also the same for both voids because the minimum charge magnitude observed from the measured PRPD pattern is almost the same for both voids since they are the same size. Simulation of 500 applied voltage cycles for this sample
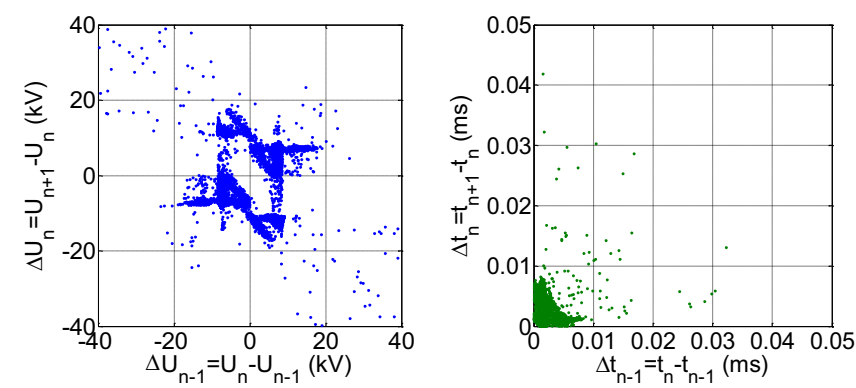

(a)
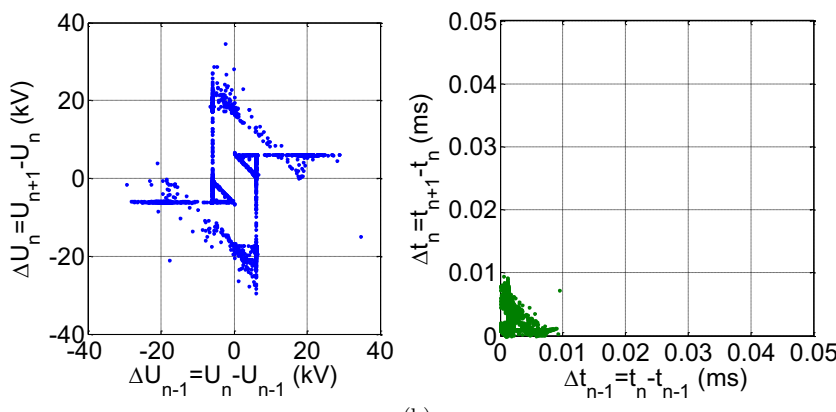

(b)

Figure 7. PSA scatter plots for PD events within sample 1; (a) measurement and $(b)$ simulation.

on $3.1 \mathrm{GHz}$ Intel(R) Core (TM) i3-2100 processor with $4 \mathrm{~GB}$ RAM took around $5 \mathrm{~h}$.

Figure 7 shows the PSA plots of PD measurement and simulation results for sample 1, which consists of scatter plots of the time difference $(\Delta t)$ and applied voltage amplitude difference $(\Delta U)$ between consecutive discharges. The simulation results have almost similar patterns with the measurement result, especially for the regions near zero, except for the regions of larger $\Delta U$ and $\Delta t$. Referring to figure 7(a), the regions of $\Delta U$ and $\Delta t$ near to zero indicate that PDs occur within two voids. In single spherical void (sample 3 ), the PSA plot of the time difference between consecutive PDs, $\Delta t$ is not very close to zero. This is due to the fact that after a PD has occurred in the void, the electric field in the void needs a certain period of time to recover to the inception field before the next PD can occur again. If $\Delta t$ is very close to zero, then 

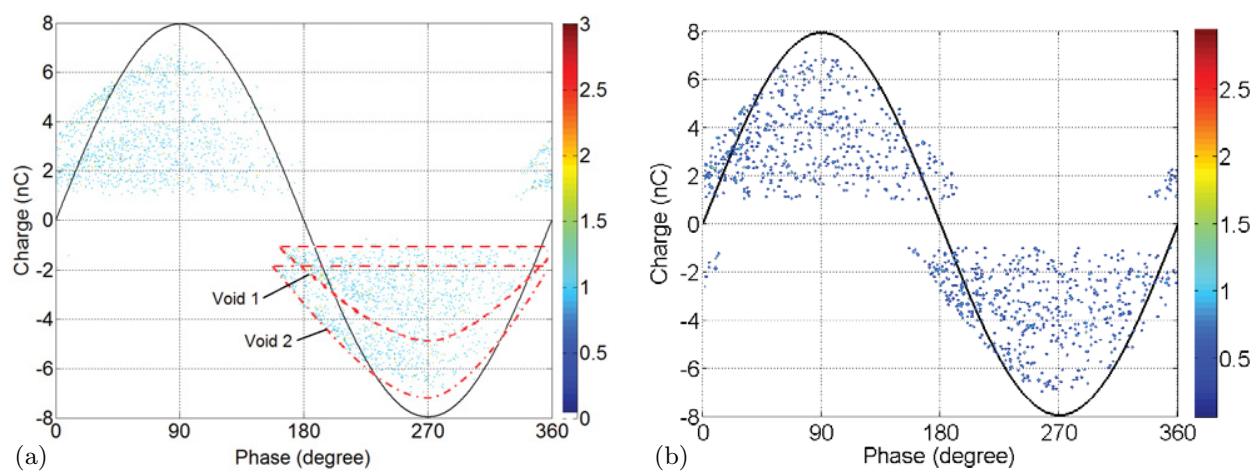

Figure 8. PRPD histograms from sample 2; $(a)$ measurement and $(b)$ simulation.

Table 5. PD data from the measurement and simulation for sample 2.

\begin{tabular}{lllll}
\hline & & \multicolumn{3}{c}{ Simulation } \\
\cline { 3 - 5 } Data & Measurement & Void 1 + Void 2 & Void 1 & Void 2 \\
\hline Total PDs per cycle & 2.1 & 2.2 & 1.1 & 1.1 \\
Total charge per cycle $(\mathrm{pC})$ & 6702 & 7198 & 2882 & 4316 \\
Mean charge $(\mathrm{pC})$ & 3191 & 3289 & 2573 & 3854 \\
Maximum charge, $q_{\max }(\mathrm{pC})$ & 7029 & 7067 & 4725 & 7067 \\
Minimum charge, $q_{\min }(\mathrm{pC})$ & 1001 & 1009 & 1009 & 1828 \\
\hline
\end{tabular}

it is likely that the next PD occurs within another void. The minimum $\Delta t$ is $8.2 \mu$ s for sample 1 while for the single void sample, it is $2.2 \mathrm{~ms}$.

\subsection{Comparison between measurement and simulation results: Sample 2}

Figure 8(a) shows the PRPD histogram of PD events obtained from PD measurement using sample 2 in table 1 . The reddashed lines show a segregation of PD patterns from two different voids, assuming they are voids 1 and 2. Sample 2 is the test object which has two spherical voids of different sizes located in an epoxy resin. From the figure, two clearly distinguished PD patterns or so-called 'tortoise-like' patterns can be observed, as opposed to the PRPD pattern from single void sample. The 'tortoise-like' patterns with larger maximum PD charge magnitude is due to PDs occurring within the larger void size (void 2). However, the number of PDs per cycle from each void cannot be determined since PDs occurring from either void cannot be distinguished. Comparison between PD measurement and simulation results for sample 2 is summarized in table 5. Both results seem to agree with each other.

The parameter values that have been used in the simulation for sample 2 are shown in table 6 . Since the size of both voids is different, the inception and extinction fields are different. The extinction field is determined from the measured minimum charge magnitude, where for this case, the electric field change in the void due to PD equals the inception field minus the extinction field. Assuming the pressure in both voids is equal, i.e. at atmospheric pressure, the inception field, $E_{\text {inc } x}$ is higher in the smaller void (void 1) according to (3). However, the extinction field, $E_{\text {ext } x}$ is higher for the larger void (void 2) than the smaller void. This is found according to the minimum
Table 6. Simulation parameters used in the model for sample 2.

\begin{tabular}{|c|c|c|c|c|}
\hline Definition & Symbol & Void 1 & Void 2 & Unit \\
\hline Void diameter & $r_{\mathrm{v} x}$ & 3.0 & 3.6 & $\mathrm{~mm}$ \\
\hline Material thickness & $h_{\text {mat }}$ & 5.4 & 5.4 & $\mathrm{~mm}$ \\
\hline Applied voltage & $U_{\text {app }}$ & 22 & 22 & $\mathrm{kV}$ \\
\hline $\begin{array}{l}\text { Higher void surface } \\
\text { conductivity }\end{array}$ & $\sigma_{\mathrm{sH} x}$ & $1 \times 10^{-12}$ & $5 \times 10^{-12}$ & $\mathrm{Sm}^{-1}$ \\
\hline Inception field & $E_{\text {inc } x}$ & 3.62 & 3.52 & $\mathrm{kVmm}^{-1}$ \\
\hline Extinction field & $E_{\text {ext } x}$ & 0.75 & 1.00 & $\mathrm{kVmm}^{-1}$ \\
\hline $\begin{array}{l}\text { Initial EGR due to } \\
\text { surface emission }\end{array}$ & $N_{\mathrm{es} 0 x}$ & 10 & 10 & $\mathrm{~s}^{-1}$ \\
\hline $\begin{array}{l}\text { EGR due to volume } \\
\text { ionization }\end{array}$ & $N_{\mathrm{ev} x}$ & 90 & 110 & $\mathrm{~s}^{-1}$ \\
\hline
\end{tabular}

PD charge magnitude from the measured PRPD patterns. Regarding the parameters related to EGR, the EGR due to volume ionization, $N_{\mathrm{ev} x}$ is higher for the larger void due to its larger volume. However, the EGR due to surface emission, $N_{\text {es } 0 x}$ is set the same for both voids because there is no obvious distinction in the number of PDs per cycle between both voids from the PRPD patterns. The void surface conductivity during charge movement along the void wall, $\sigma_{\mathrm{sH} x}$ is set higher for the larger void. This is done in order to reproduce the maximum PD charge magnitude observed from the measured PRPD patterns for each void. Simulation of 500 applied voltage cycles for this sample on 3.1 GHz Intel(R) Core (TM) i3-2100 processor with 4 GB RAM took around $6 \mathrm{~h}$.

From figure 8(a), most PDs occur after a certain time delay when the inception field has been exceeded. This is due to lower EGR, which reduces the probability of PD occurrence. Thus, it results in a lower number of PDs per cycle, which can be seen by the low density of PD distribution pattern from both voids.

The PD phase distributions of PRPD pattern in figure $8(a)$ are shown in figure 9 . From the $H_{n}$ distribution, there are more 

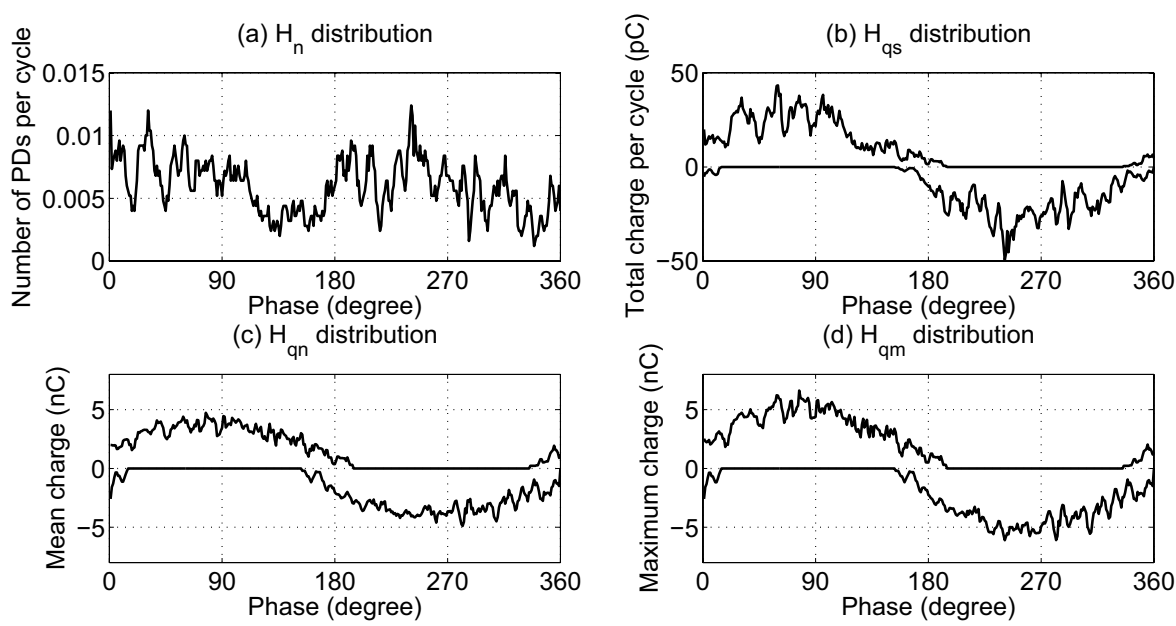

Figure 9. Phase distribution of PD activity measurement from sample 2.
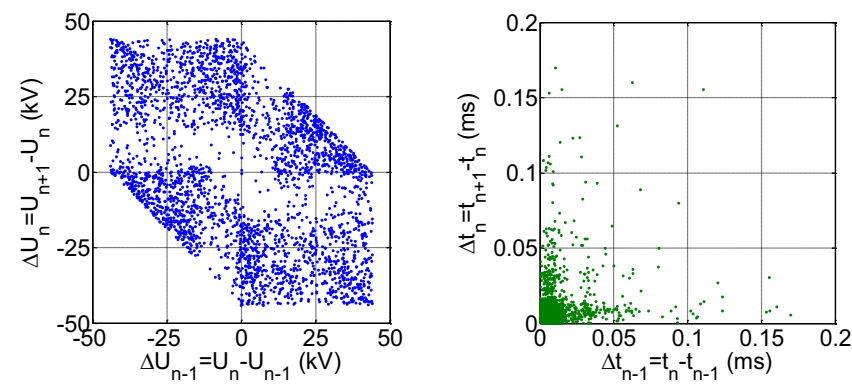

(a)
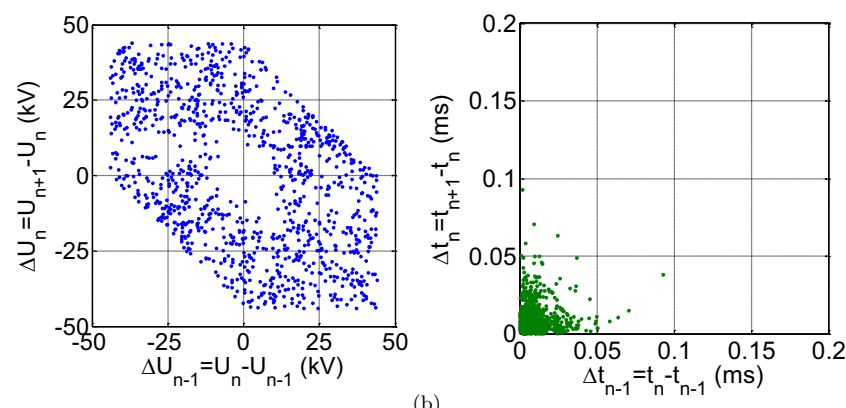

(b)

Figure 10. PSA scatter plots for PD events within sample 2; $(a)$ measurement and $(b)$ simulation.

PDs occurring in the first and third quadrants than the second and fourth quadrants of the applied voltage. This is because the inception field is exceeded most of the time within the first and third quadrants. From the $H_{\mathrm{qs}}$ distribution, the total charge per cycle is larger at the phase nearer to $90^{\circ}$ and $270^{\circ}$ because most PDs occur with maximum magnitude around these phases. The $H_{\mathrm{q} n}$ distribution shows the mean charge magnitude increases towards the phase of $90^{\circ}$ and $270^{\circ}$ and decreases after these phases. The $H_{\mathrm{qm}}$ distribution is also similar to $H_{\mathrm{qn}}$ distribution. The maximum charge magnitude follows the applied voltage magnitude, where the maximum PD charge magnitude occurs during the peak magnitude of the applied voltage.

The PSA plots of PD measurement and simulation results for sample 2 are shown in figure 10. Both results seem to have similar patterns. From figure 10(a), again, the regions of $\Delta U$ and $\Delta t$ near to zero indicate that PDs occur within two voids, as compared to the PSA plots from the single void sample. The
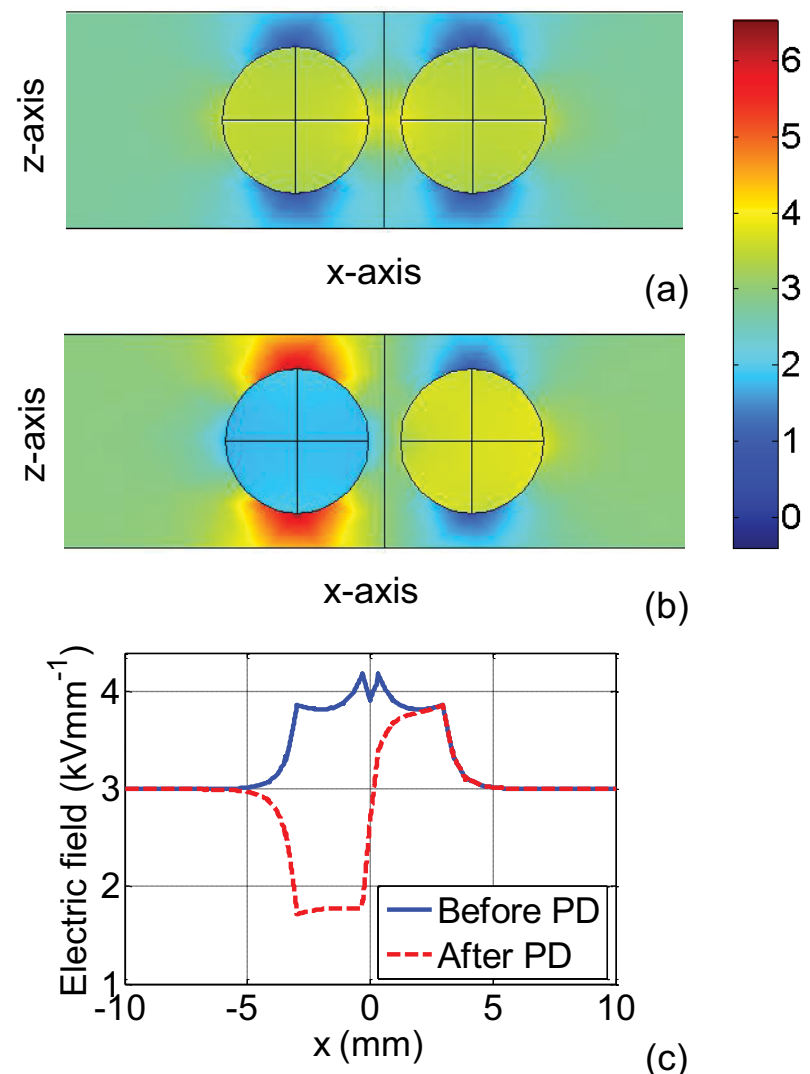

(c)

Figure 11. Electric field distribution (in $\mathrm{kV} \mathrm{mm}^{-1}$ ) in the FEA model geometry for sample 1 ; before $(a)$ and after $(b)$ a PD occurs in void 1 and $(c)$ the electric field line plot across the middle section of the sample.

PSA patterns between the measurement and simulation results are within reasonable agreement to each other. The minimum $\Delta t$ is $10.2 \mu$ s for sample 2 .

\subsection{Electric field distribution from the FEA model}

Figures 11 and 12 show the electric field distribution in the FEA model geometry for samples 1 and 2 before and after a PD occurrence. The parameters used are shown in tables 4 and 6. 

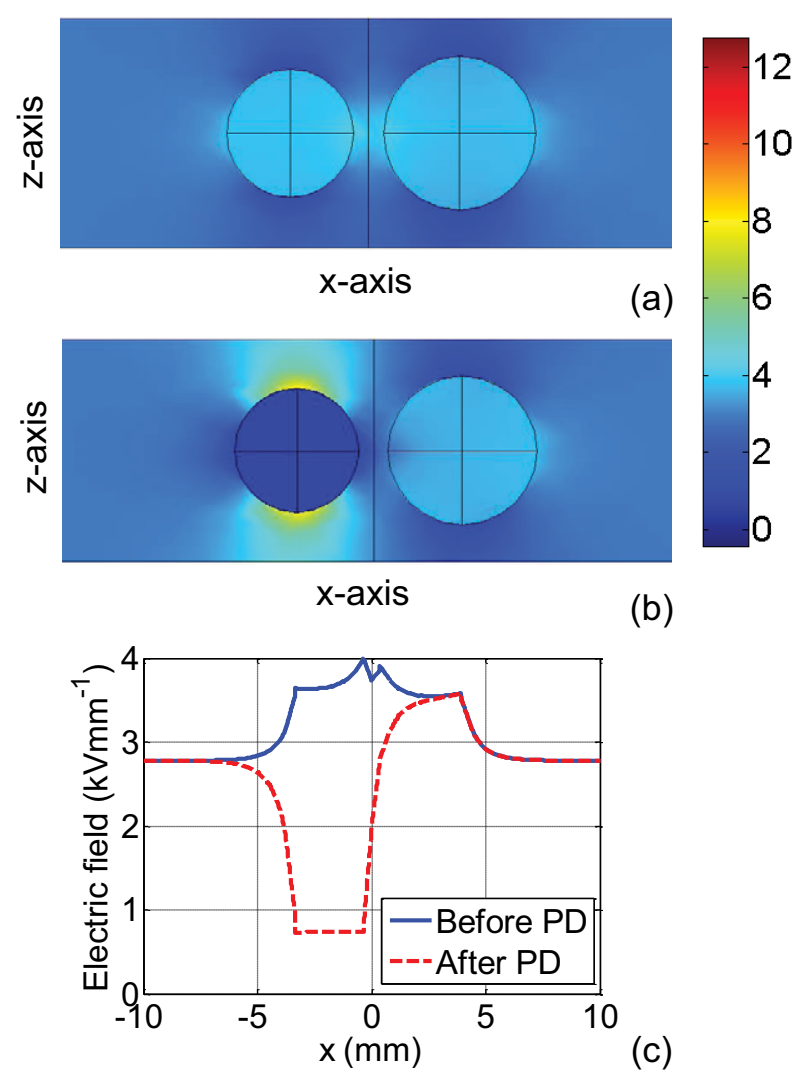

Figure 12. Electric field distribution (in $\mathrm{kV} \mathrm{mm}^{-1}$ ) in the FEA model geometry for sample 2; before $(a)$ and after $(b)$ a PD occurs in void 1 and $(c)$ the electric field line plot across the middle section of the sample.

From the simulation, before a PD occurs, the field in the void region that is nearest to the other void is highest. This electric field behaviour is found to be similar to the condition when part of the void surface is very close to the insulation material boundary. Hence, a void that is closely located to another void can be said to behave as an insulation material boundary to that void. After a PD occurs in one of the voids, say void 1, which causes the field in void 1 to decrease, the field in the other void, say void 2, is also slightly reduced (referring to figures $11(c)$ and $12(c)$ ). Therefore, when two voids are closely located to each other, the field in each void is influenced by the other.

\subsection{Simulation of electric field magnitudes versus time}

The simulations of the electric field magnitudes against time of the applied voltage for samples 1 and 2 obtained using FEA model are shown in figure 13. The simulations use parameters as defined in tables 4 and 6 . Again, it can be seen that when a PD occurs in one of the voids, the electric field in the other void decreases slightly. Hence, the total EGR of each void will also be affected by the other because the total EGR depends on the electric field in the void. This will in turn affect the probability of a PD occurrence and the number of PDs per cycle.

\section{Conclusion}

A three-dimensional (3D) model geometry of two spherical voids within a homogeneous dielectric material has been
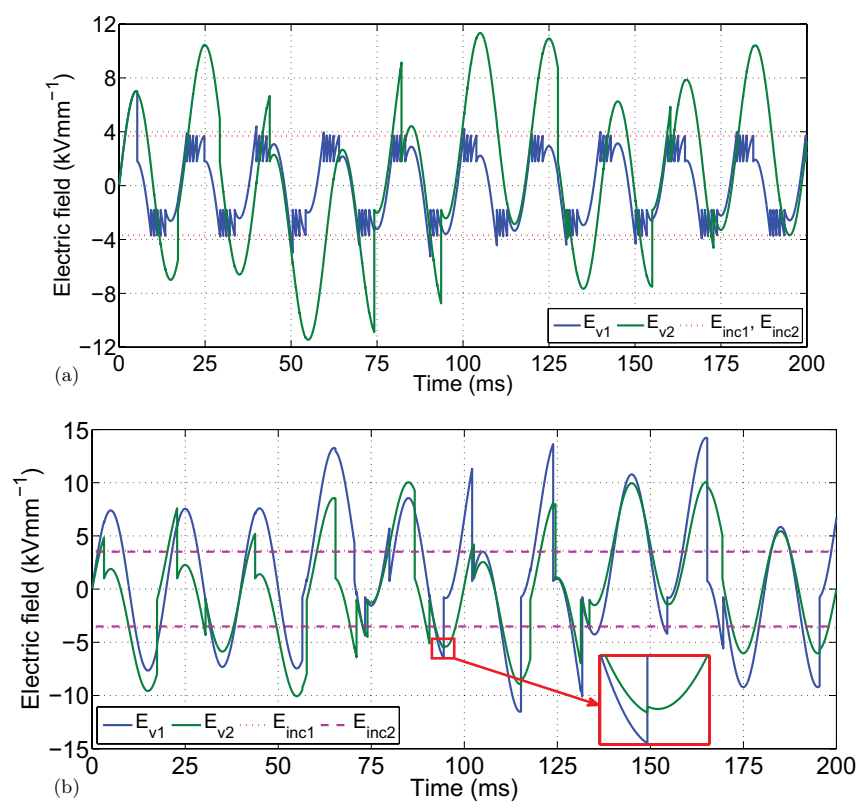

Figure 13. Simulation of electric field distribution against time of the applied voltage from the FEA model for; $(a)$ sample 1 and $(b)$ sample 2 .

developed using FEA software. The model has been used to simulate PD activity within two voids. From the measurement results of PRPD patterns, PD patterns from two different voids can be clearly seen. However, some individual PD occurrences cannot be clearly distinguished between the two voids because the patterns from both voids are overlapping each other. PD activities occurring in two voids have been verified further through PSA scatter plots. It was found that the patterns from PDs occurring within two voids are clearly different from PDs occurring within single void. The appearance of near zero value in the PSA scatter plots shows that PDs occur within two voids as opposed to single void, where there is no near zero value found in its PSA plots. Therefore, PRPD patterns and PSA scatter plots can clearly show PDs occurring within single and multiple voids in a dielectric material.

Comparison between measurement and simulation results was made and they are within reasonable agreement to each other. From the simulation of PD activities that has been performed, parameters affecting PD behaviour in each void have been identified. They are the electron generation rate due to surface emission and volume ionization, void surface conductivity and the inception and extinction fields. The inception and extinction fields mainly affect the minimum PD charge magnitude, the void surface conductivity affects the maximum PD charge magnitude and the electron generation rate mainly affects the number of PDs per cycle. However, these parameters are inter-related to each other. A change in one of the parameters will affect the simulation results, which causes other parameters needing to be changed until the simulation results match the measurement data. Thus, sensitivity analysis was used to determine the simulation parameters. Through simulation, PDs occurring from two voids have been successfully modelled. This allows the number of PDs per cycle that occur from each void, which 
cannot be determined directly from the measurement results, to be obtained.

\section{Acknowledgments}

The authors thank the Tony Davies High Voltage Laboratory, University of Southampton, UK for providing the test facilities and technical knowledge and Malaysian Ministry of Higher Education (MOHE) for supporting this work through MOHE HIR research grant (H-16001-D00048).

\section{References}

[1] Zhang C H 2003 IEEE Trans. Dielectr. Electr. Insul. $10312-19$

[2] Boggs S A 1990 IEEE Electr. Insul. Mag. 6 11-16, $19-20$

[3] Ruay-Nan W and Chien-Kuo C 2011 IEEE Trans. Pwr Deliver. 26 1585-91

[4] Wu K, Suzuoki Y and Dissado L A 2004 J. Phys. D: Appl. Phys. 37 1815-23

[5] Heitz C 1999 J. Phys. D: Appl. Phys. 32 1012-23

[6] Niemeyer L 1995 IEEE Trans. Dielectr. Electr. Insul. 2 510-28
[7] Gutfleisch F and Niemeyer L 1995 IEEE Trans. Dielectr. Electr. Insul. 2 729-43

[8] Schifani R, Candela R, and Romano P 2001 IEEE Trans. Dielectr. Electr. Insul. 8 589-97

[9] Forssen C and Edin H 2008 IEEE Trans. Dielectr. Electr. Insul. 15 1610-6

[10] Morshuis P, Cavallini A, Montanari G C, Puletti F and Contin A 2000 Int. Conf. on Properties and Applications of Dielectric Materials (Xi'an, China) pp 304-9

[11] Illias H, Chen G, and Lewin P L 2011 IEEE Trans. Dielectr. Electr. Insul. 18 432-43

[12] Seung-Ik J, Doe-Sung S, Do-Hong Y, Key-Man H and Min-Koo H 1995 Int. Conf. on Conduction and Breakdown in Solid Dielectrics (Leicester, UK) pp 398-402

[13] Hossam-Eldin A A, Dessouky S S, El-Mekkawy S M and El-Aal R A A 2007 Conf. on Electrical Insulation and Dielectric Phenomena (Vancouver) pp 792-6

[14] Ghourab M E and El-Makkawy S M 1994 Conf. on Electrical Insulation and Dielectric Phenomena (Arlington, TX) pp 155-60

[15] Illias H, Chen G, and Lewin P L 2011 IEEE Electr. Insul. Mag. 27 38-45

[16] Illias H, Chen G, and Lewin P L 2011 J. Phys. D: Appl. Phys. 44245202

[17] MacAlpine J M K and Zhang C H 2003 IEEE Trans. Dielectr. Electr. Insul. 10 506-13

[18] Rainer P and Farhad B 2002 J. Phys. D: Appl. Phys. 3525 